\title{
Induced Pluripotent Stem Cells, A Slippery Slope for Neurodegenerative Disease Modelling?
}

\author{
Kuldip S. Sidhu* \\ Stem Cell Laboratory, School of Psychiatry, Faculty of Medicine, 408, Wallace Wurth Building, University of New \\ South Wales, NSW, Australia
}

\begin{abstract}
The recent breakthrough in reprogramming somatic cells has invigorated the prospect that disease mechanisms that underpin various human diseases particularly the neurodegenerative disorders could be unravelled by using the disease-specific pluripotent stem cells. A number of studies have demonstrated that such disease-specific induced pluripotent stem cell (iPSC) could be generated relatively easy. Some recent studies have substantiated the utility of this technology in describing the initial characterization of neurodegenerative patient-derived iPSC as a proof of concept. However, as it is becoming evident now that the cell type of origin influences the molecular and functional properties of derived iPSC. The indications that reprogramming may erase the cell memory also raises the question if the disease phenotype may not be correctly represented or also erased in iPSC unless coaxed by further perturbation in vitro culture conditions. Other associated difficulties in iPSC research such as culture variability, selective adaptation of such cultures and the lack of robust protocols to generate homogeneous population of desired cell type may have compounding affects in the use of these cells in disease modelling. Unless these issues are addressed properly the prospects of iPSC in disease modelling may remain a slippery slop.
\end{abstract}

Keywords: Induced pluripotent stem cells, disease modelling, neurodegenerative diseases, epigenetic, reprogramming.

\section{INTRODUCTION}

Neurodegenerative diseases represent a cohort of diverse symptoms in which specific neuronal subtypes degenerate and die either sporadically or due to familial links. Majority of the cases are sporadic. These diseases constitute more than $14 \%$ of the global burden in neurological ailments [1], and with rise in life expectancy, their incidence is also expected to rise. Current pharmacological interventions only alleviate certain symptoms and can produce serious side effects. Stem cell-based therapies for neurodegenerative disorders are attractive, given the limited regenerative capacity of the mammalian central nervous system. Several preclinical studies have generated encouraging data showing that stem cells and their derivatives can improve function and mitigate neurodegeneration in a variety of experimental models of neurological disorders [2].

Recent advances in stem cell biology have raised the prospect that perhaps the difficulty in understanding the disease mechanisms that underpin neurodegenerative disorders probably due to the limited access to human nerve cells - could finally be overcome with the availability of pluripotent stem cells. These human stem cells can, in principle, be differentiated into any cell types, including degenerating neural cells. To date, such pluripotent stem cells can be derived by two methods: the derivation of human emb-

*Address correspondence to this author at the Stem Cell Laboratory, School of Psychiatry, Faculty of Medicine, 408, Wallace Wurth Building, University of New South Wales, NSW, Australia; Tel: +61 29385 3938; Fax:+61 29385 3938; E-mail: k.sidhu@unsw.edu.au ryonic stem cells (hESC) from the inner cell mass of preimplantation embryos that have been discarded from in vitro fertilization clinics, and the conversion of somatic cells, such as skin fibroblasts, into iPSC by genetic manipulations (Fig. 1). Routinely, iPSC are generated using a cocktail of over expressed transcription factors transferred to the recipient cells by transfection or viral vector infection; improved methods that avoid genetic modification of donor cells are under development [3]. Both hESC and iPSCs have been derived in author's laboratory including those from patients with an early onset of Alzheimer's disease [4-6]. We also compared hESC and iPSC [6] by microarray gene expression profiling and have demonstrated that these cell types are similar albeit not identical. They share the ability to self renew indefinitely while maintaining the potential to differentiate into cells from lineages of all germ layers (Fig. 2). The advantage of iPSC over hESC is the possibility of modelling neurological diseases by deriving pluripotent cells directly from somatic cells of affected individuals. Pluripotent stem cells have already been derived from individuals carrying inherited defects [7-10], as well as from those with other common neurological disorders such as sporadic Parkinson's disease $[11,12]$, thus offering the unprecedented opportunity to gain insights into disease mechanisms and to search for new drugs using human disease-specific cell lines. iPSC may also provide the chance to obtain a renewable source of healthy cells to treat neurological disorders, a view that is supported by the demonstration that human pluripotent cell-derived dopaminergic neurons alleviate some of the locomotor abnormalities seen in a rat model of Parkinson's disease [11, 13]. 


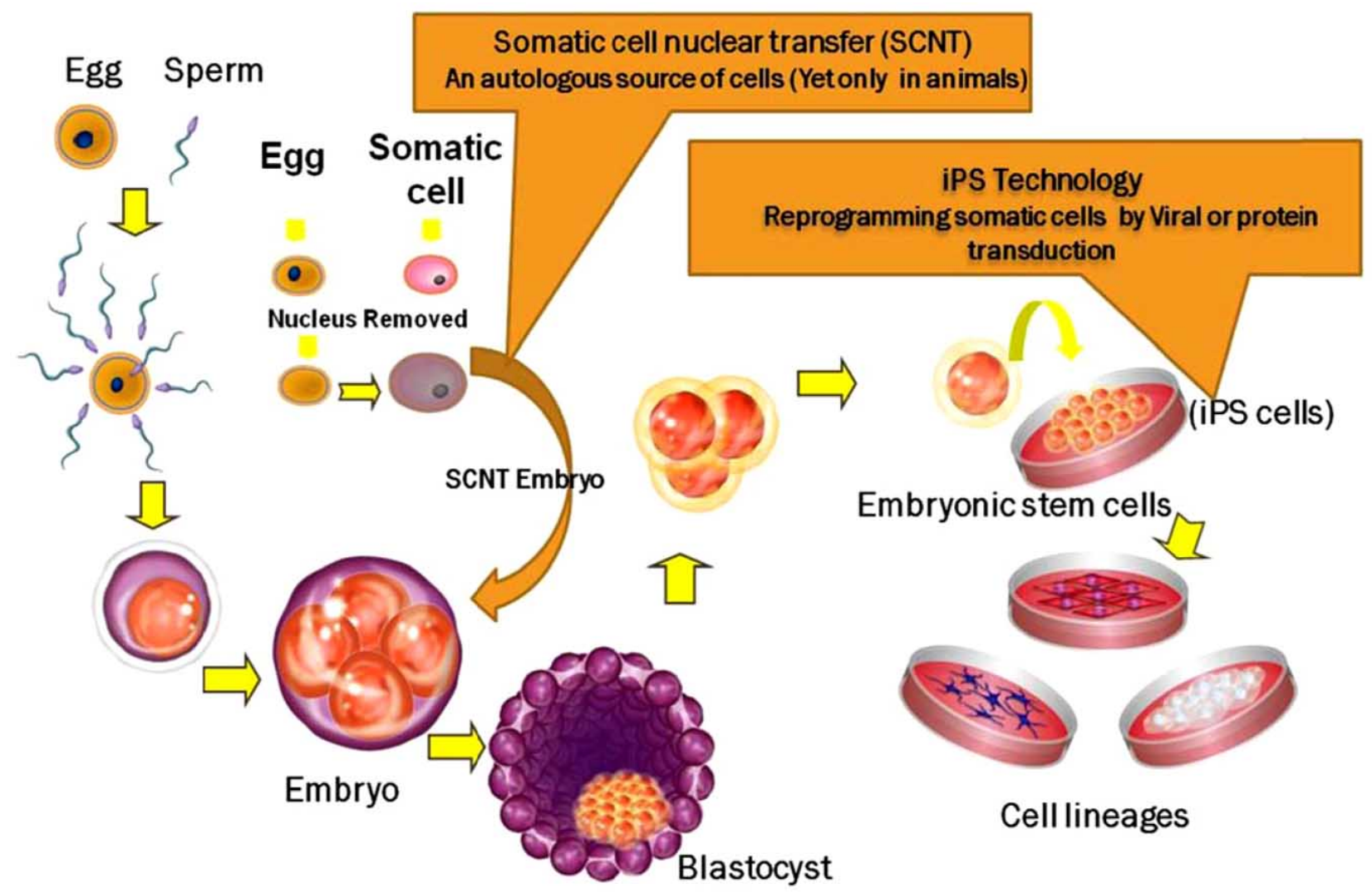

Fig. (1). The pluripotent human embryonic stem cells (hESCs) are derived after fertilisation from day 5 embryo called blastocyst. At this stage it consists of about 200 cells of which 30-40 cells constitute the inner cell mass (ICM) and the others form the trophectoderm that eventually forms the placenta. hESCs are derived by dissecting out ICM and growing these on feeder. These cells are pluripotent and form all lineages derived from three germ layers. In animals an autologous source of such cells have been produced by SCNT but not yet in humans by simply removing sperm with a somatic cells. Recently somatic cell can be reprogrammed to its pluripotent state by simply transducing with four pluripotent genes, Oct4, Sox2, Klf4, c-Myc either by viral or protein transduction (iPS technology). Such pluripotent cells called as induced pluripotent stem (iPS) cells though can generate different lineages derived from three germ layers for future regenerative medicine, but has limitations because of transgenes and these as such are currently being developed for drug discovery.

\section{PRE-CLINICAL MARKERS}

The clinical symptoms of neurodegenerative diseases such as Alzheimer's (AD) are due to extensive neuronal loss. Neuro protective strategies instigated at the onset of clinical symptoms could conceivably reduce further loss but are unlikely to impact on the existing disability. Ideally identifying patients in a preclinical or prodromal state where they retain relatively normal function is very desirable. However preclinical detection will only be useful if we have effective treatments.

Prospective studies of older person cohorts with comprehensive neuropsychiatric evaluation, Neuro imaging, and medical profiling and post mortem confirmation may offer our best opportunity for the retrospective detection of potential preclinical markers [14-16]. Alternatively animal models, offer reasonable phenocopies of $\mathrm{AD}$, or at least the monogenic forms of it, but suffer because comparative genomic and anatomical differences are manifest most in relation to the human brain and experiments are generally carried out in inbred populations. There is a definite need for alternative humanised model systems, particularly to explore the gene $\mathrm{x}$ environmental interactions that are postulated to underline the pathogenesis of complex neurodegenerative diseases.

\section{STEM CELL TECHNOLOGY AND IPS CELLS}

The study of human development, ageing and disease is limited by a lack of model systems that can reproduce the precise sequence and timing of cellular and molecular events. Pluripotent cells including the recent iPSC that now can be derived from patient skin [12] have the inherent ability to recapitulate human development in vitro. Therefore, these cells offer a huge potential to follow disease progression in a dish and gain valuable insight into the pathognomic of that disease, discover new prognostic biomarkers, and ensure a continuous supply of afflicted cell types for drug discovery. Two recent studies $[8,10]$ describe the initial characterisation of neurodegenerative disease patient-derived iPSC lines as proof of concept for the utility of this technology.

\section{PATIENT-DERIVED CELL LINES AS DISEASE MODELS}

Patient derived cell models can potentially overcome the issues with animal models and decrease the natural history of $\mathrm{AD}$ from years to months [17]. Such patient-derived cell lines provide tremendous opportunities for explorative casecontrol studies and subsequent preclinical drug discovery 


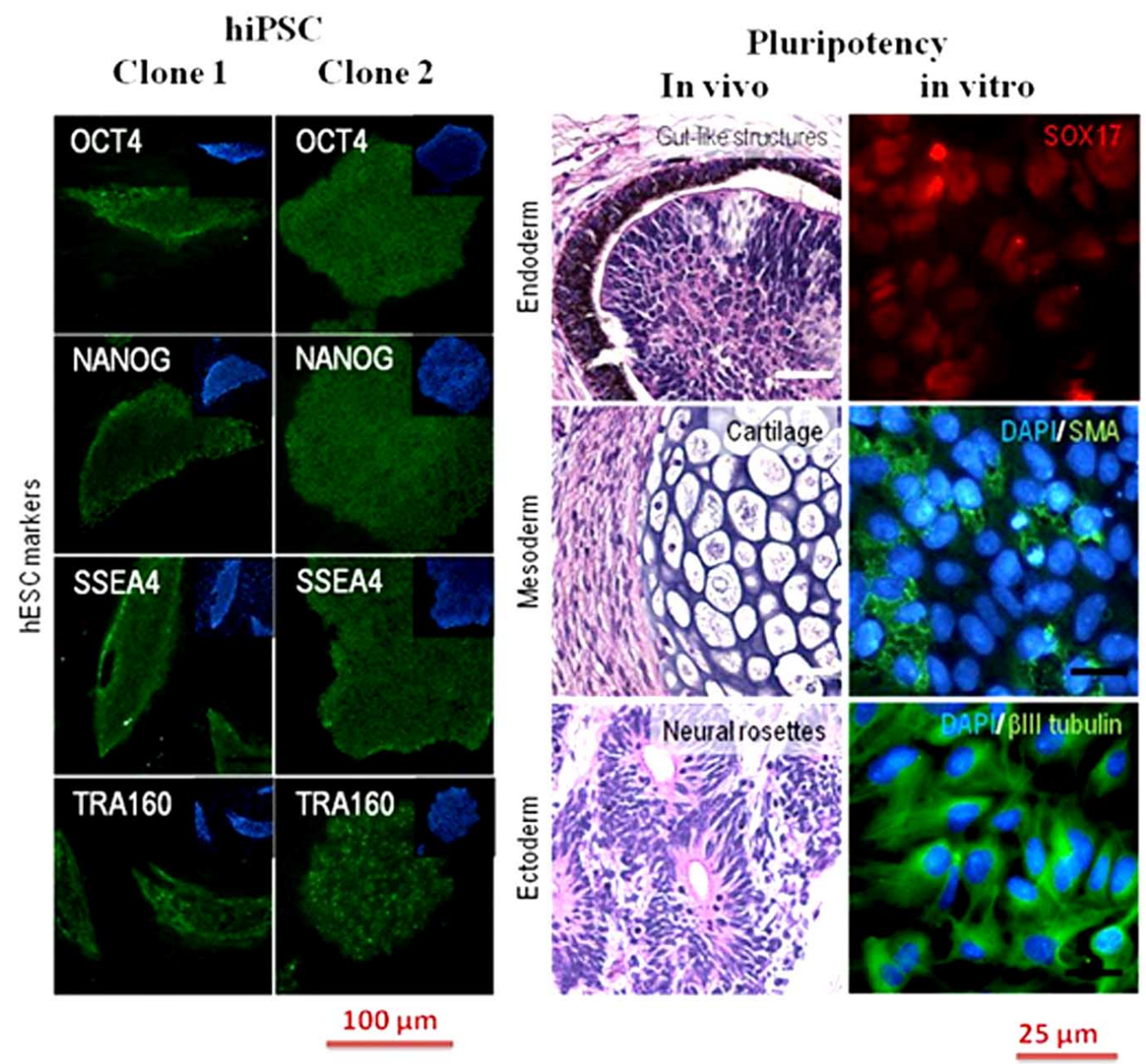

Fig. (2). Human induced pluripotent stem cell (hiPSC) clones expressing pluripotent markers (left panel) and indication of pluripotency in these clones under both in vivo and in vitro conditions (right panel) from Chung et al. [6].

[18]. Cell models of amyotrophic lateral sclerosis [19], Parkinson's disease [20], and spinal muscular atrophy have been developed and in the case of the latter, cured in vitro [21]. Recently iPSC have been derived from adult human skin with specific diseases $[11,12,22]$. Current reports using iPSC to model genetic diseases like spinal muscular atrophy and familial dysautonomia have shown disease-specific phenotypes that may be important for modelling and drug discovery $[9,10]$. Importantly these cell lines represent the genomic background of each individual, a variable that would otherwise be impossible to model outside of clinical trials. iPSC derived from affected individuals provide a unique opportunity to gain insights into the neurobiology of neurodegenerative diseases. Disease modelling with iPSC will be a three part process, first the derivation of diseasespecific iPSC, secondly to differentiate these cells effectively to cell lineage of choice like neurons, oligodendrocytes and astrocytes that hopefully express disease phenotype and thirdly to use these derived lineage-specific cells for high through put screening to model the disease and functional analyses if used for therapeutics down the track (Fig. 3). None of these steps is easy and also we need to recapitulate in vitro key aspects of neuronal degeneration in a time frame that is conducive to pathophysiological studies and, eventually, to drug screening. Transgenic animal models have taught us a great deal about disease process in some of the diseases but question remains whether therapeutically significant insights will be gained from the study of animal models. In this context, iPSC cells derived from humans with inherited neurological disorders may provide invaluable tools for elucidating the mechanisms by which the diseasecausing gene products kill neurons in the human cellular context and at endogenous levels of expression. However, there are number of issues as below that we need to address to before realising the full potential of stem cell technology.

\section{IPSCS AS SLIPPERY SLOPE FOR DISEASE MODELLING}

Critics of stem cell disease models suggest that casecontrol differences will merely represent the impact of the disease rather than an underlying genetic susceptibility or physiological difference. Ageing and disease effects in chronic conditions such as AD are likely to be mediated via epigenetic effects on gene expression. As iPS cell technology erodes epigenetic signatures, this cell memory may be lost, both in the iPS cells themselves and their descendents as reported recently in iPSC derived from fragile X syndrome patient [23]. This 'ground/naïve state' appears akin to the putative inherent or embryological differences between an AD-susceptible and unaffected individual. Alternatively one could maintain the cell memory by pursuing the culture 
of adult stem cells without a pluripotent stage [24, 25] but here the problem would be separating disease 'cause' from 'effect'. Similarly the direct reprogramming of fibroblasts to neurons is likely to retain this 'cell memory' [26].

Derivation of iPS cells from people with neurodegenerative diseases and their differentiation into clinically relevant cell types are only the first steps on the road to successful therapy. As many neurodegenerative disorders are adult onset, identification of disease-related phenotypes in short-term in vitro settings might be a particular challenge, unless it turns out that the ontogenic age of the iPSC derivatives matches that of the donor rather than that of embryonic cells We could also expect that the differences in naïve iPSC from patients to manifest themselves through differentiation processes that could be traced by following a developmental paradigm. There are now the bases to recapitulate the life history of the disease through subtle perturbations with AD-related exposures, and create differentiable phenotypes in vitro. Of possible relevance to this issue is the observation that, thus far, disease-related cellular phenotypes have been observed in iPS models only of developmental neuropathologies: spinal muscular atrophy and familial dysautonomia $[9,10]$. In contrast to the models of neurodevelopmental disorders, no disease-related phenotypes have been thus far reported in iPSC from adult-onset diseases such as ALS or Parkinson's disease. Perhaps here the disease phenotype may never manifest itself under basal cell culture conditions, but it may be revealed by challenging the neural cells with stressors such as nitrogen or oxygen reactive species, proinflammatory factors or even toxins as explained above. Identification of these relevant stressors eliciting early neuronal phenotypes in models of adult-onset neurologic diseases will therefore be important milestones for future research.

Another important factor that may have compounding effect is the erasing of the cellular memory with artificial reprogramming mechanisms used in creating such iPSC in vitro. An example of this is reported recently by Urbach et al. [23] that highlighted in fragile X mutation, the FMR1 gene is expressed in undifferentiated cells but undergoes transcriptional silencing after ESC differentiation and a significant difference exist between FX-ESCs and FX-iPSCs with regard to their expression of the FMR1 gene. The mutated FMR1 gene is expressed in FX-ESCs and transcriptionally silenced upon differentiation, whereas in FX-iPSCs the FMR1 locus remains inactive and is not reset by the reprogramming process to the transcriptionally active state. It is thus possible that other disorders related to epigenetic defects, including triplet repeat and imprinting disorders, may likewise evade the reprogramming process. However, differentiation of FX-iPSCs into neurons may facilitate the study of FMR1 in neural cells. Until a deeper understanding of the potential differences between iPSCs and hESC is delineated, the study of both iPSC from patients and human ESCs carrying the same mutation (either from PGD embryos or by genetic manipulation) might, whenever possible, be the optimal approach to model human genetic disorders through cell culture. In contrast, a recent study by Tchieu and coworkers [27] indicated that female human iPSCs retain an inactive X Chromosome and that has critical implications for clinical applications and disease modelling, and could be exploited for studies of X-linked diseases $[23,28]$ and for a unique form of gene therapy for X-linked diseases.

Once disease-specific phenotypes are identified and translated into robust cell-based assays, the most consequential use of iPS cells derived from affected individuals will be the screening of candidate drugs by, for example, high-throughput platforms (Fig. 3). Such efforts at drug discovery will be greatly facilitated by the virtually unlimited supply of pluripotent cells and their derivatives. Another potential advantage in using iPS technology is that the new drugs will have already been tested on human cells, which may facilitate the identification of better therapies and accelerate their translation to the clinic.

According to Polo and co-workers [29] genetically matched iPSC retain a transient transcriptional and epigenetic memory of their cell of origin at early passage, which can substantially affect their potential to differentiate into embryoid bodies and different hematopoietic cell types. These molecular and functional differences are lost upon continuous passaging, These data also serve as a cautionary note for ongoing attempts to recapitulate disease phenotypes in vitro using patient-specific, early-passage iPSC lines, as the epigenetic, transcriptional and functional 'immaturity' of these cells might confound the data obtained from them. Further elucidation of the molecular indicators of fully reprogrammed iPSCs should help in the establishment of standardized iPSC lines that can be compared with confidence in basic biological and drug discovery studies.

The continuous presence of trans genes in iPSC harbour the risk of modifying the target genome and also interfere with the subsequent differentiation process in such cells. Therefore recent protein-based hiPSC technology offers a new and potentially safe method for generating patientspecific stem cells. This system completely eliminates genome manipulation and DNA transfection, resulting in human iPSCs suitable for drug discovery, disease modelling, and future clinical translation [3, 30, 31]. Similarly other studies suggest that it may be possible to replace and/or further reduce the number of factors required for reprogramming [32-34]. To minimize/avoid chromosomal disruption, adenovirus and plasmid transfection have been successfully used to generate iPSCs in the mouse system [35, 36]. Also, Thomson and his colleagues reported generation of hiPSCs by transfection with nonintegrating episomal vectors [37]. In addition, piggyback transposon [35, 38] and Cre-recombinase excisable viruses [22] have been used to generate hiPSCs. While the transgenes can be excised by inducible gene expression once reprogramming is established [22, 38, 39], residual sequences and chromosomal disruptions may still result in harmful alterations that could pose clinical risks.

Last but not the least, most of the studies on neurodegenerative diseases have focussed mostly only on the neural part of the brain. However, the involvement of non-neuronal cells, such as astrocytes or microglia, in the overall neurodegenerative process is increasingly recognized. Studies on chimeras, composed of mutant and wild-type cells or animals in which genetic defects were genetically attenuated in subsets of neurons or non-neuronal cells, argue for a significant non-autonomous contribution to the disease processes [40]. Subsequently, a series of in vivo and in vitro studies has 

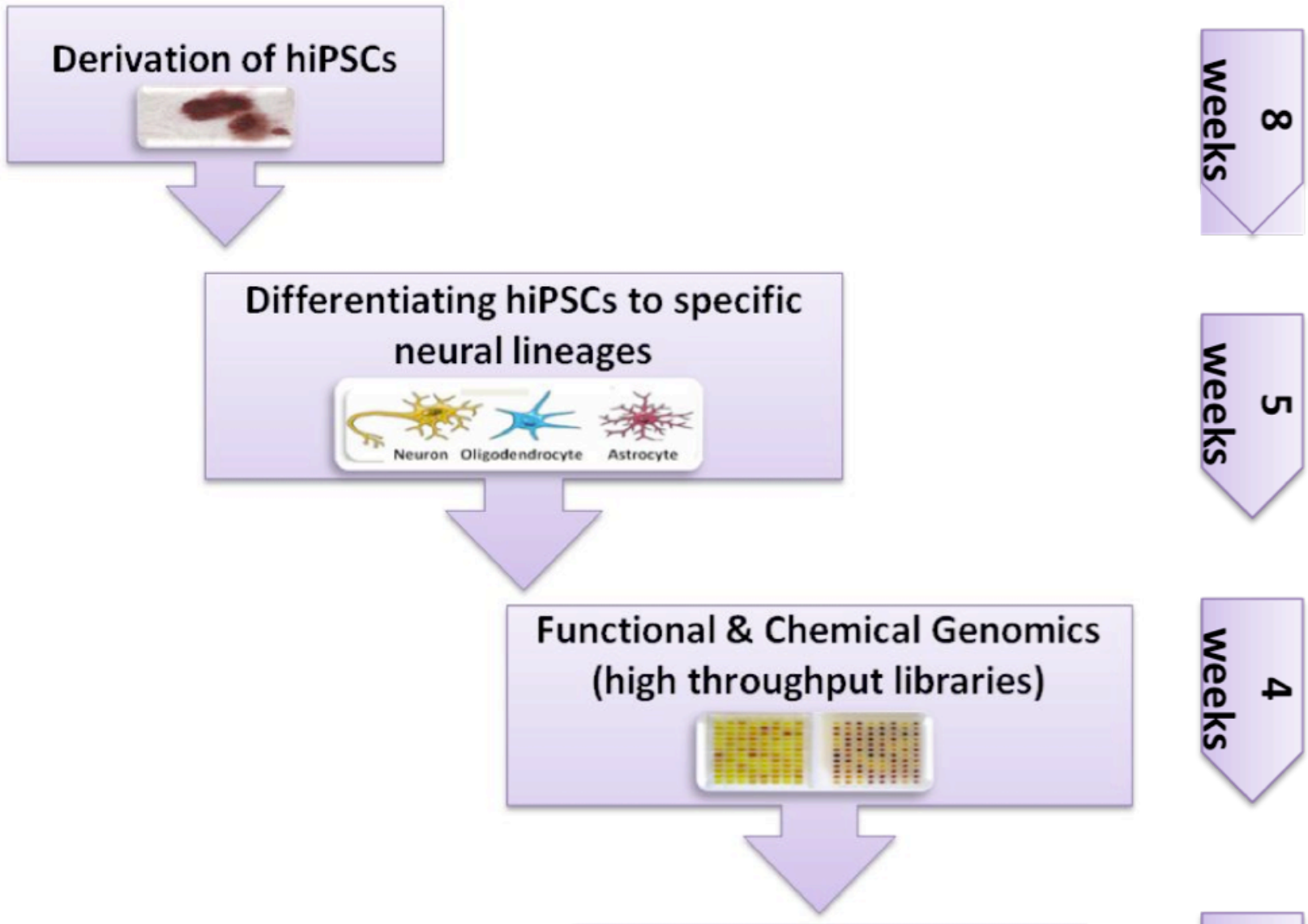

MECHANISMS OF DISEASE Gain-Loss of-function screens
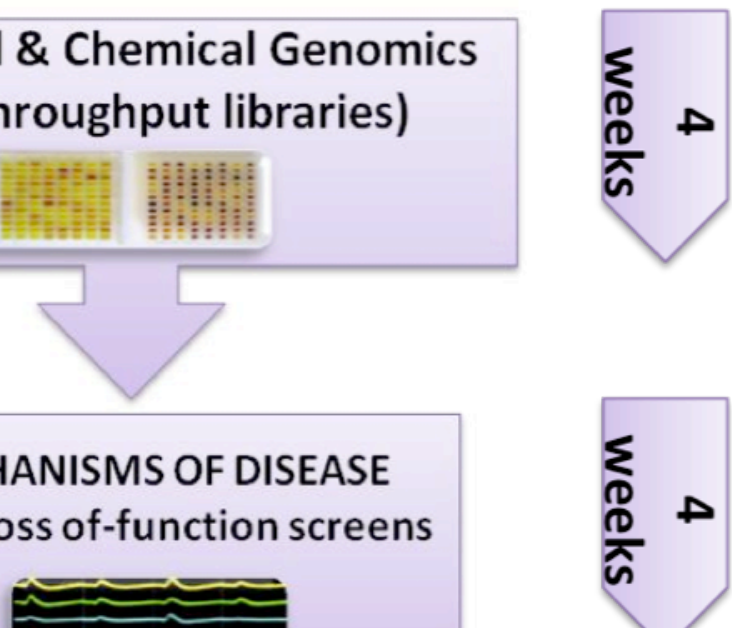

Fig. (3). Human induced pluripotent stem cells (hiPSCs) as Cellular Models for Neurodegenerative Diseases as a three step phenomenon i.e. derivation, differentiation and functional annotation of hiPSCs and their derived neural progenies by high throughput assays with a time line.

provided compelling evidence that mutant non-neuronal cells can transmit the neurodegenerative phenotype to wild-type neighbouring neurons [41-44]. This has been achieved in mouse models of ALS, whereby astrocytes carrying a mutated form of the superoxide dismutase-1 (SOD1) gene were shown to mediate a toxic activity that kills both wild-type and mutant SOD1 mouse motor neurons as well as human ES cell-derived motor neurons.

\section{REFERENCES}

[1] World Health Organization. Neurological Disorders: Public Health Challenges. Geneva: World Health Organization 2006.

[2] Lindvall O, Kokaia Z. Stem cells in human neurodegenerative disorders-time for clinical translation? J Clin Invest 2010; 120: 2940.

[3] Han J, Sachdev PS, Sidhu KS. A Combined Epigenetic and NonGenetic Approach for Reprogramming Human Somatic Cells. Plos ONE 2010; 5: e12297.

[4] Sidhu KS, Ryan JP, Tuch BE. Derivation of a new hESC line, endeavour-1 and its clonal propagation. Stem Cells Develop 2008; 7: $34-45$.
[5] Sidhu KS, Ryan JP, Lees JG, Tuch BE. Derivation of a new human embryonic stem cell line, endeavour-2 and its characterization. In vitro Cells Develop Biol 2010; 46: 269-75.

[6] Chung H, Lin RCY, Logan GJ, Alexander IE, Sachdev PS, Sidhu KS. Human induced pluripotent stem cells derived under feederfree conditions display unique cell cycle and DNA replication genotypes. Stem Cell Develop 2011; (Under Publication).

[7] Sermon KD, Simon C, Braude P, Viville S, Borstlap J, Veiga A. Creation of a registry for human embryonic stem cells carrying an inherited defect: joint collaboration between ESHRE and hESCreg. Hum Reprod 2009; 24: 1556-60.

[8] Dimos JT, Rodolfa KT, Niakan KK, et al. Induced pluripotent stem cells generated from patients with ALS can be differentiated into motor neurons. Science 2008; 321: 1218-21.

[9] Lee G, Papapetrou EP, Kim H, et al. Modelling pathogenesis and treatment of familial dysautonomia using patient-specific iPSCs. Nature 2009; 461: 402-6.

[10] Ebert AD, Yu J, Rose FF Jr, et al. Induced pluripotent stem cells from a spinal muscular atrophy patient. Nature 2009; 457: 277-80.

[11] Wernig M, Zhao JP, Pruszak J, et al. Neurons derived from reprogrammed fibroblasts functionally integrate into the fetal brain and improve symptoms of rats with Parkinson's disease. Proc Natl Acad Sci USA 2008; 105: 5856-61.

[12] Park IH, Arora N, Huo $\mathrm{H}$, et al. Disease-specific induced pluripotent stem cells. Cell 2008; 134: 877-86. 
[13] Rodriguez-Gomez JA, Lu JQ, Velasco I, et al. Persistent dopamine functions of neurons derived from embryonic stem cells in a rodent model of Parkinson disease. Stem Cells 2007; 25: 918-28.

[14] Cherbuin N, Reglade-Meslin C, Kumar R, et al. Risk factors of transition from normal cognition to mild cognitive disorder: the PATH through Life Study. Dement Geriatr Cogn Disord 2009; 28 : 47-55.

[15] Jorm AF, Butterworth P, Anstey KJ, et al. Memory complaints in a community sample aged 60-64 years: associations with cognitive functioning, psychiatric symptoms, medical conditions, APOE genotype, hippocampus and amygdala volumes, and white-matter hyperintensities. Psychol Med 2004; 34: 1495-506.

[16] Sachdev PS, Lammel A, Trollor JN, et al. A comprehensive neuropsychiatric study of elderly twins: the Older Australian Twins Study. Twin Res Hum Genet 2009; 12: 573-82.

[17] Dauer W, Przedborski S. Parkinson's disease: mechanisms and models. Neuron 2003; 39: 889-909.

[18] Marchetto MC, Muotri AR, Mu Y, Smith AM, Cezar GG, Gage FH. Non-cell-autonomous effect of human SOD1 G37R astrocytes on motor neurons derived from human embryonic stem cells. Cell Stem Cell 2008; 3: 649-57.

[19] Wichterle H, Lieberam I, Porter JA, Jessell TM. Directed differentiation of embryonic stem cells into motor neurons. Cell 2002; 110: 385-97.

[20] Ming GL, Song H. Adult neurogenesis in the mammalian central nervous system. Annu Rev Neurosci 2005; 28; 223-50.

[21] Mizuseki K, Sakamoto T, Watanabe K, et al. Generation of neural crest-derived peripheral neurons and floor plate cells from mouse and primate embryonic stem cells. Proc Natl Acad Sci USA 2003; 100: $5828-33$.

[22] Soldne F, Hockemeyer D, Beard C, et al. Parkinson's disease patient-derived induced pluripotent stem cells free of viral reprogramming factors. Cell 2009; 136: 964-77.

[23] Urbach A, Bar-Nur O, Daley GQ, Benvenisty N. Differential modeling of fragile $\mathrm{X}$ syndrome by human embryonic stem cells and induced pluripotent stem cells. Cell Stem Cell 2010; 6: 407-11.

[24] Murrell W, Wetzig A, Donnellan M, et al. Olfactory Mucosa is a Potential Source for Autologous Stem Cell Therapy for Parkinson's Disease. Stem Cells 2008; 26: 2183-92.

[25] Valenzuela MJ, Dean SK, Sachdev P, Tuch BE, Sidhu KS. Neural precursors from canine skin: a new direction for testing autologous cell replacement in the brain. Stem Cells Dev 2008; 17: 1087-94.

[26] Vierbuchen T, Ostermeier A, Pang ZP, Kokubu Y, Südhof TC, Wernig M. Direct conversion of fibroblasts to functional neurons by defined factors. Nature 2010; 463: 1035-41.

[27] Tchieu J, Kuoy E, Chin MH, et al. Female Human iPSCs Retain an Inactive X Chromosome. Cell Stem Cell 2010; 7(3): 329-42.

[28] Agarwal S, Loh YH, McLoughlin EM, et al. Telomere elongation in induced pluripotent stem cells from dyskeratosis congenita patients. Nature 2010; 464: 292-6.
[29] Polo JM, Liu S, Figueroa ME, et al. Cell type of origin influences the molecular and functional properties of mouse induced pluripotent stem cells. Nat Biotechnol 2010; 28: 848-55.

[30] Kim D, Kim C, Moon J, et al. Generation of Human Induced Pluripotent Stem Cells by Direct Delivery of Reprogramming Proteins. Cell Stem Cell 2009; 4: 1-5.

[31] Huangfu D, Osafune K, Maehr R, et al. Induction of pluripotent stem cells from primary human fibroblasts with only Oct4 and Sox2. Nat Biotechnol 2008; 26: 1269-75.

[32] Li W, Wei W, Zhu S, et al. Generation of rat and human induced pluripotent stem cells by combining genetic reprogramming and chemical inhibitors. Cell Stem Cell 2009; 4: 16-19.

[33] Shi Y, Desponts C, Do JT, Hahm HS, Scholer HR, Ding S. Induction of pluripotent stem cells from mouse embryonic fibroblasts by Oct 4 and Klf4 with small-molecule compounds. Cell Stem Cell 2008; 3: 568-74.

[34] Kaji K, Norrby K, Paca A, Mileikovsky M, Mohseni P, Woltjen K. Virus-free induction of pluripotency and subsequent excision of reprogramming factors. Nature 2009; 458: 771-5.

[35] Okita K, Nakagawa M, Hyenjong H, Ichisaka T, Yamanaka S. Generation of mouse induced pluripotent stem cells without viral vectors. Science 2008; 322; 949-53.

[36] Yu J, Hu K, Smuga-Otto K, et al. Human induced pluripotent stem cells free of vector and transgene sequences. Science 2009; 324; 797-801.

[37] Woltjen K, Michael IP, Mohseni P, et al. PiggyBac transposition reprograms fibroblasts to induced pluripotent stem cells. Nature 2009; 458: 766-70.

[38] Stadtfeld M, Nagaya M, Utikal J, Weir G, Hochedlinger K. Induced pluripotent stem cells generated without viral integration. Science 2008; 322: 945-9.

[39] Lobsiger CS, Cleveland DW. Glial cells as intrinsic components of non-cell-autonomous neurodegenerative disease. Nat Neurosci 2007; 10: 1355-60.

[40] Ballas N, Lioy DT, Grunseich C, Mandel G. Non-cell autonomous influence of MeCP2-deficient glia on neuronal dendritic morphology. Nat Neurosci 2009; 12: 311-17.

[41] Custer SK, Garden GA, Gill N, et al. Bergmann glia expression of polyglutamine-expanded ataxin-7 produces neurodegeneration by impairing glutamate transport. Nat Neurosci 2006; 9: 1302-31.

[42] Nagai M, Re DB, Nagata T, et al. Astrocytes expressing ALSlinked mutated SOD1 release factors selectively toxic to motor neurons. Nat Neurosci 2007; 10: 615-22.

[43] Zhu S, Wurdak H, Wang J, et al. A small molecule primes embryonic stem cells for differentiation. Cell Stem Cell 2009; 4; 416-26.

[44] Wichterle H, Predborski S. What can pluripotent stem cells teach us about neurodegenerative diseases? Nature Neurosciences 2010; 13: $800-4$.

(C) Kuldip S. Sidhu; Licensee Bentham Open.

This is an open access article licensed under the terms of the Creative Commons Attribution Non-Commercial License (http://creativecommons.org/licenses/by$\mathrm{nc} / 3.0 /$ ), which permits unrestricted, non-commercial use, distribution and reproduction in any medium, provided the work is properly cited. 